\title{
PROVISION OF END-TO-END DELAY GUARANTEES IN WAVELENGTH-ROUTED OPTICAL BURST-SWITCHED NETWORKS
}

\author{
Ignacio de Miguel ${ }^{1}$, Eugene Kozlovski ${ }^{2}$, Polina Bayvel ${ }^{2}$ \\ ${ }^{1}$ E.T.S.I. de Telecommunicación, University of Valladolid, Campus Miguel Delibes, Camino del \\ Cementerios/n, 47011 Valladolid, Spain \\ ignmig@tel.uva.es \\ ${ }^{2}$ Optical Networks Group, Department of Electronic and Electrical Engineering, UCL \\ (University College London), Torrington Place, London WCIE 7JE, UK. \\ \{ekozlovs, pbayvel\} @ee.ucl.ac.uk
}

\begin{abstract}
Alstract: In Wavelength-Routed Optical Burst-Switched Networks (WR-OBS), lightpaths are dynamically established for the transmission of bursts (aggregation of packets in edge routers) through a bufferless optical core. In this paper, we show how WR-OBS can provide end-to-end delay guarantees, and introduce methods to achieve fairness in the network. The conditions under which WR-OBS bring operational advantages when compared to wavelength-routed optical networks (WRONs) are identified using an example of a real network and taking into account the diameter of the network and the number of wavelengths required.
\end{abstract}

Key words: Optical networks, optical burst switching, end-to-end delay, routing and wavelength assignment, fairness

\section{INTRODUCTION}

A Wavelength-Routed Optical Burst Switched (WR-OBS) network architecture has been recently proposed and analysed as a promising packet network model to provide guaranteed latencies [1-3]. Optical burst-switched (OBS) networks [4-5] consist of optical core nodes connected to electronic edge routers, where packets are buffered according to their destination and class of service to form a burst, which is transmitted through the core 
network. Thus, the OBS approach combines the processing and buffering capabilities of electronics at the network edge with the advantage of optical WDM transport and wavelength routing in the core. In contrast to the earlier OBS approaches [4,5], WR-OBS proposes the use of dynamic lightpaths for the transmission of bursts. Each of the previous works on WR-OBS [1-3,6,7] has focused on one of the design sub-problems, assuming ideal scenarios for the others. In this paper, we evaluate the operational benefits of WR-OBS achievable in the design of optical transport networks assuming realistic network conditions, and compare WR-OBS with wavelength-routed optical networks (WRONs) [8]. Although either centralised or distributed algorithms can be used in WR-OBS, a centralised control scheme is assumed in this work to identify the conditions under which it satisfies the QoS requirements. The design conditions under which WR-OBS guarantee bounded end-to-end delays for $100 \%$ of packets are identified, and methods to provide fairness are introduced. A network is considered to be fair if the blocking probability of a connection between a source-destination pair is independent of the location of these nodes in the network [9].

\section{BURST AGGREGTION METHODS}

In the WR-OBS architecture, packets are buffered in edge routers, according to their destination and class of service, for burst aggregation. Associated with each buffer there is a timer, and according to it, after a predefined time period $(T)$ from the arrival of the first packet of the burst, the process of requesting and establishing a unidirectional lightpath between the source and destination edge routers has to be initiated. In the centralised version, the edge router sends a request to a control node, which solves the dynamic routing and wavelength assignment (DRWA) problem and sends commands to cross-connects to establish the lightpath. The edge router must wait for an acknowledgement confirming that the lightpath has been established to be able to transmit the burst. Two methods to aggregate bursts, Limited-Burst Size (LBS) and Unlimited-Burst Size (UBS), have been proposed [3]. In LBS, the arrival of the acknowledgement to the edge router determines the end of the aggregation process of the packets into a burst. Then, new packets arriving to the buffer during the burst transmission are not aggregated into this burst, but must wait for another lightpath to be established for their transmission. In UBS, new packets arriving at the buffer after the reception of the acknowledgement are considered as part of the current burst, and hence, the transmission process only finishes when the buffer is empty, at which point the lightpath is deleted. Typical burst sizes are in the range of tens of milliseconds when LBS is used, and tens or 
hundreds of ms - depending on the traffic load - when UBS is used. In [3], we suggested that UBS consistently performed with lower blocking probability than LBS. This result is further explored here together with techniques for setting timers to achieve fairness.

\section{END-TO-END DELAY GUARANTEES WITH FAIRNESS}

One of the key parameters when providing QoS in networks is the endto-end delay, which is defined as the time elapsed from the arrival of a packet to the source edge router until it is delivered to the destination edge router. In a WR-OBS, the end-to-end delay of a packet can be decomposed in three terms - the time elapsed for the packet in the buffer at the source edge router $\left(t_{b u f f e r}\right)$, the transmission delay of the packet $\left(t_{t x}\right)$ and the propagation delay from the source to the destination edge router $\left(t_{p r o p, s-d}\right)$. The transmission delay is usually not significant when compared to the other terms; note that the most common $\mathbb{P}$ packet sizes are 44 and 1500 bytes [10], so for $10 \mathrm{Gbit} / \mathrm{s}$ transmission $t_{t x}=35.2 \mathrm{~ns}$ and $1.2 \mu \mathrm{s}$ respectively, while $t_{\text {buffer }}$ and $t_{\text {prop,s-d }}$ are in the range of milliseconds (take for example the propagation delay through $200 \mathrm{~km}$ of fibre, which is approximately $1 \mathrm{~ms}$ ).

Previous work on WR-OBS was focused on the analysis of the edge delay $\left(t_{\text {edge }}\right)[1-3,6,7]$, defined as the waiting time for the first packet of a burst from its arrival to the buffer until its transmission. Assuming that the peak bit-rate per source-destination pair and class of service does not exceed the capacity - bit-rate - of a lightpath, then the delay elapsed by any packet in the buffer will be equal or lower than $t_{\text {edge, }}$ that is, $t_{\text {buffer }} \leq t_{\text {edge }}$. Therefore, guaranteeing a maximum end-to-end delay $\left(\Delta_{e e, \max }\right)$ for all the packets is equivalent to guaranteeing that the end-to-end delay of the first packet of every burst is below that value. The end-to-end delay for such a packet $\left(\Delta_{e e, b}\right)$ is defined by $\Delta_{e e, b}=t_{\text {edge }}+t_{t x}+t_{\text {prop }, s-d} \approx t_{\text {edge }}+t_{\text {prop },-d-d}$.

In a WR-OBS with centralised control, $t_{\text {edge }}$ depends, in turn, on:

- the value of the timer $(T)$ set to determine request sending time,

- twice the propagation delay from the source edge router to the control node $\left(2 t_{p r o p, s-c t r l}\right)$-request and acknowledgement propagation,-

- the processing delay in the control node, which includes the calculation time of the algorithm $\left(t_{c}\right)$ and the queuing delay $\left(t_{q}\right)$, - note that these are non-deterministic values,

- and the delay required for tuning the laser in the source node and for the reconfiguration of the cross-connects in the source-destination path $\left(t_{\text {tuning }}\right)$. Therefore, the following relationship must hold, 


$$
T+2 t_{\text {prop }, s-c t r l}+t_{q}+t_{c}+t_{\text {tuning }}+t_{\text {prop }, s-d} \leq \Delta_{e e, \max } .
$$

The location of the nodes plays an important role on the performance of dynamic connections to other nodes. Note that $t_{p r o p, s-c t r l}$ depends on the relative placements of the source edge router and the control node in the network, and $t_{\text {propss-d }}$ depends on the location of the source and destination nodes, on the DRWA strategy, and on the network resource availability. This information must be taken into account in order to provide a fair service, so that all source-destination pairs (from now on, $s-d$ pairs) have the same guarantees independently of their location in the network. We denote the physical location-dependent terms in equation (1) by $\Delta_{p h, s d}=2 t_{p r o p, s-c t r l}+$ $t_{p r o p, s-d}$, and we refer to this parameter as the location-dependent delay. Note that during the dynamic operation of the network, in general, different routes will be allocated for the transmission between a $s-d$ pair. The minimum value of $\Delta_{p h, s d}$ is obtained when the shortest delay path is employed, then the minimum location-dependent delay for a $s-d$ pair is $\Delta_{p h, s d \text {,min }}=2 t_{\text {prop,s-ctrl }}+$ $t_{\text {prop,s-d,min, }}$ where $t_{\text {prop,s-d,min }}$ is the propagation delay through such a path.

\subsection{Control node and strategies for faimess provision}

In the centralised architecture, the functions of the control node are to receive requests, search for a route and a wavelength, and transmit acknowledgements/rejection messages to source nodes as well as commands to optical cross-connects for the establishment of lightpaths. A novel component in the control node, the request scheduler, was described as a method to improve the efficiency of DRWA algorithms and optimise the wavelength requirements while bounding the latency in the control node [6]. As shown here, it allows further advantages as controlling the end-to-end delay while providing faimess.

In this work we consider a classless network, so only one queue is required in the control node to store requests. The request scheduler selects the next one to be processed according to a certain policy. We employ a number of policies based on Earliest Deadline First (EDF), as it is the optimal scheduling discipline to support bounded delay services [11]. Once a request is selected, it is passed to the DRWA block, which performs a search for a route and wavelength. If it is found, it is checked whether the acknowledgement time to the source node, the tuning delay and the propagation delay to the destination node are low enough so that $\Delta_{e e, \max }$ is not exceeded. Otherwise - if it is exceeded or if no route was found, - the request is added to a temporal set of unsuccessfully processed requests, where it remains until any lightpath is released. At that point, the request is 
moved back to the queue, so that it can be selected again for another attempt by the DRWA block. When a request has stayed in the control node so long that even if it were assigned the shortest delay route to the destination node $\Delta_{e e, \max }$ would be exceeded, it is deleted from the control node, and a rejection message is submitted towards the source node of that request. Note that rejection of requests is not due to blocking of the DRWA algorithm but to being unable to provide an end-to-end delay below $\Delta_{e e, \max }$. A parameter related to the request scheduler is the maximum scheduling time $\left(t_{\text {sched,max }}\right)$, which is defined as the maximum delay allowed for a request to stay in the control node (including queuing delay and unsuccessful stays in the DRWA block, but excluding the calculation time of one attempt - and assuming the worst case $t_{c, \max }$ ) while satisfying the maximum allowable end-to-end delay. Thus,

$$
\Delta_{e e, b} \leq T+\Delta_{p h, s d, \text { min }}+t_{c, \text { max }}+t_{\text {tuning }}+t_{\text {sched,max }}=\Delta_{e e, \max } .
$$

The scheduling time has a threefold function: (a) to allocate time for queuing, (b) to allow for multiple connection attempts if a lightpath cannot be established at the first attempt in the DRWA block, and (c) to provide extra time so that longer paths than the shortest delay one can be allocated.

As was previously mentioned, the propagation delays can vary depending on the location of the control node and of the nodes involved in the transmission. In this work, we study two different main scenarios to adjust the timers and to implement the EDF policy in the control node. With the first strategy (scenario A) no distance information is used at edge routers (but it may be employed in the control node), while with the second (scenario B), the location-dependent delay is used at edge routers. The aim of these strategies is to provide as much fairness as possible. For each class of service, faimess is defined as the provision of guaranteed maximum endto-end delay with uniform blocking probability (due to exceeding this delay) for all $s-d$ pairs.

\subsubsection{Scenario $\mathbb{A}$ : Edge routers without knowledge of $\Delta_{p h, s d, \text { min }}$}

In this scenario, all edge routers set their timers to the same value $T$, independently on their distance to the control node and independently on their distance to the destination node. By doing so, the maximum scheduling time will be different for different $s-d$ pairs. Since the equality in (2) must hold, $s-d$ pairs with low $\Delta_{p h, s d, \text { min }}$ will have a high value of $t_{s c h e d, \max }$, and vice versa. This clearly leads to an unfair network as "easy" connections are allocated more scheduling time $\left(t_{\text {sched,max }}\right)$ than "difficult" ones, and hence, 
the latter connections will suffer higher blocking probabilities than the former. In order to introduce fairness, the request scheduler can use information on the delay experienced by the requests and on distance between involved nodes to select the next request to process. Depending on how much of this information is used, a number of policies can be defined:

A.1 Select the request that has stayed longer in the control node. As no additional information is used, this policy results in unfair treatment of the connections between more distant node pairs.

A.2 Select the request with highest elapsed edge delay $\left(t_{\text {edge elapsed }}\right)$. The elapsed edge delay is the waiting time of the first packet of the burst.

A.3 Select the request that has the highest $t_{\text {edge_elapsed }}+t_{\text {prop,s-ctrl }}+t_{\text {tuning. }}$.

A.4 Select the request with highest $t_{\text {edge_elapsed }}+t_{\text {prop,s-crrl }}+t_{\text {tuning }}+t_{\text {prop,s-d,min }}$.

To use one of these three policies, the control node be provided with information on the elapsed edge delay when the request is received, which must be included with the request by the source edge router. This information is then updated by the control node, taking into account the propagation delay of the request, and the time spent within the control node.

\subsubsection{Scenario $\mathbb{B}$ : $\mathbb{E} d g e$ routers with knowledge of $\Delta_{p h, s d, \text { min }}$}

Edge routers are aware of their distance to the control node, and of their minimum distance to other edge routers, so if an edge router is going to transmit to an edge router close to it, it will send the request to the control node later than if it wants to transmit to an edge router which is far from it. Each timer in the network is adjusted so that $T+\Delta_{p h, s d, \text { min }}$ is equal for all of them. By doing so, the maximum scheduling time allocated for each sourcedestination lightpath request will be the same one independently on their location of the network. The scheduler selects the next request to be processed as that with the highest value of $t_{\text {edge elapsed }}+t_{\text {prop,s-ctrl }}+t_{\text {tuning }}+$ $t_{\text {prop,s-d,min, }}$ which in this case is equivalent to selecting the request which has spent the longest time within the control node.

\section{CASE STUDY: THE NSFNET AS A WR-OBS}

By way of illustration of the above points and to explore whether WR-OBS networks have performance advantages compared to WRONs, we analyse the centralised WR-OBS architecture for the NSFNet topology (Figure 1) by means of simulation (all results are shown with 95\% confidence intervals). The NSFNet consists of 14 nodes and 21 bidirectional links. We assume there is one edge router per core router. Each edge router has 13 buffers - one per destination - where constant bit-rate (CBR) traffic 
is being aggregated into bursts. The size of the buffers will be calculated below. We assume that all $s-d$ pairs have the same traffic load (defined as the ratio between the input bit-rate at each buffer and the bit-rate of a lightpath -which was set to $10 \mathrm{Gbit} / \mathrm{s}-)$. The location of the control node was selected so that the average propagation delay from the nodes of the network to it was minimised. When doing so, it was determined that the optimal location is Pennsylvania (PA). The control topology was set by establishing shortest-delay paths from every node to the control node (Figure 1). The control topology could be implemented physically by reserving a wavelength or by using a parallel low capacity link. We assume that cross-connects require $1 \mathrm{~ms}$ to be reconfigured, and tunable lasers in edge routers also require a tuning time of $1 \mathrm{~ms}$ to switch between wavelengths.

As shown in [8], for this physical topology 13 wavelengths are sufficient to map a full logical topology with 1 lightpath-worth of demand between every $s-d$ pair. Therefore, a WR-OBS with 13 wavelengths would give no operational resource reduction when compared to a WRON (under the assumption that the peak input bit-rate to each buffer does not exceed the capacity of a lightpath). We study the case of 12 wavelengths (plus a control channel) as a limiting scenario.

A classless network is studied, but strict requirements are set so that real time services are guaranteed. In order to allow videoconference services, the end-to-end delay should be below $100 \mathrm{~ms}$ [12]. We reserve $10 \mathrm{~ms}$ for processing at end nodes (for instance for compression issues) and therefore the target for the network is to provide a guaranteed end-to-end delay of at most $90 \mathrm{~ms}$ for $100 \%$ of packets. The target maximum average burst blocking probability (due to being unable to provide an end-to-end delay below $90 \mathrm{~ms}$ ) is set to $10^{-4}$.

Under the assumptions of traffic, lightpath capacity and delays considered, each buffer at edge routers requires $108 \mathrm{MB}(90 \mathrm{~ms}$ of data arriving at highest input bit-rate, $10 \mathrm{Gbit} / \mathrm{s}$ ) to ensure no packet loss due to buffer overflow, so that packet loss is only due to rejected lightpath requests.

The minimum location dependent delay for a connection between nodes CA2 and NY is $60.975 \mathrm{~ms}$. Therefore, even assuming no queuing delay in the control node, and that the shortest-delay path is found at the first attempt in the DRWA block, the minimum end-to-end delay will be $61.975 \mathrm{~ms}$ (as the tuning delay is $1 \mathrm{~ms}$ ). In contrast, the connection between PA and NJ (or NY), can have an end-to-end delay as low as $2.575 \mathrm{~ms}$. This fact shows the asymmetry of the network in terms of propagation delays. Note that $\Delta_{p h, s d \text {,min }}$ imposes a limit on the maximum diameter of the network, or equivalently on the minimum end-to-end delay that can be guaranteed in a network. For instance, a WR-OBS architecture is not viable for NSFNet if the aim is to guarantee end-to-end delays for all packets below $60.975 \mathrm{~ms}$. 
We employ the dynamic routing and wavelength assignment algorithm AUR-EXHAUSTIVE [13] since it is an efficient one as shown in [13,14]. Moreover, it gives preference to routes with lower number of hops, which is a good idea from the point of view of delay (although it is not the optimal one). The average calculation time is assumed to follow a $\operatorname{Beta}(0,0.2,5,5)$ distribution, which gives values bounded by 0 and $0.2 \mathrm{~ms}$ (so, $t_{c, \max }=0.2$ $\mathrm{ms}$ ), and with average $0.1 \mathrm{~ms}$.

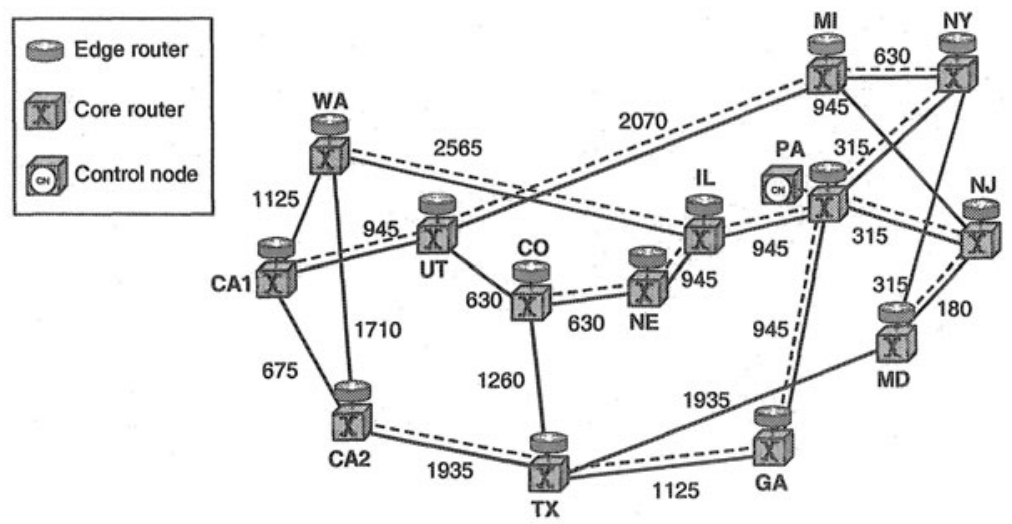

Figure 1. Centralised WR-OBS architecture applied to the NSFNET topology. We assume one edge router per core router. The control node is set in Pennsylvania (PA), and the dashed lines show the control topology. Distances between nodes are shown in $\mathrm{km}$ [15].

\subsection{Analysis of the scenarios for providing fairmess}

There is an average burst blocking probability associated with each $s-d$ pair, which should be uniform in a fair network. The problem of studying each pair separately is that very long simulations are required in order to analyse low blocking probabilities. Therefore, we first study the global average burst blocking probability, which is defined as the number of rejected requests divided by all requests received by the control node. Then we study fairness for high blocking probabilities (around $10^{-2}$ ) analysing the blocking probability associated to every $s$ - $d$ pair separately.

\subsubsection{Scenario A: Edge routers without knowledge of $\Delta_{p h, s d, \text { min }}$}

Tests have been done for LBS and UBS burst aggregation schemes using the four policies explained for the scheduler under this scenario. The best results on blocking probability are obtained for the fourth policy (A.4), which is the one that employs more information about delays. Evidence on this will be shown later. Results are plotted in Figures 2.a (LBS) and 2.b 
(UBS). In both cases, the best results on blocking probability are obtained when timers are set to $T=0 \mathrm{~ms}$. When LBS is employed a traffic load of up to nearly 0.2 can be carried by the network guaranteeing a maximum end-toend delay of $90 \mathrm{~ms}$ and with a global average burst blocking probability below $10^{-4}$. The network can carry a higher traffic load - up to 0.3 with the same guarantees - when using UBS. Moreover, for all loads UBS obtains lower blocking probabilities than LBS. This confirms the suggestion given in [3], where we showed that UBS uses resources more efficiently than LBS.

\subsubsection{Scenario $B$ : Edge routers with knowledge of $\Delta_{p h, s d, \text { min }}$}

Figures 2.c and 2.d show results on blocking probability for LBS and UBS respectively. In scenario $A$, all $s-d$ pairs set their timers to the same value of $T$. Now, different pairs have different values of the timer, but all of them have the same value for $t_{\text {sched,max }}$. For that reason, results are plot as a function of the maximum scheduling time instead of $T$. 

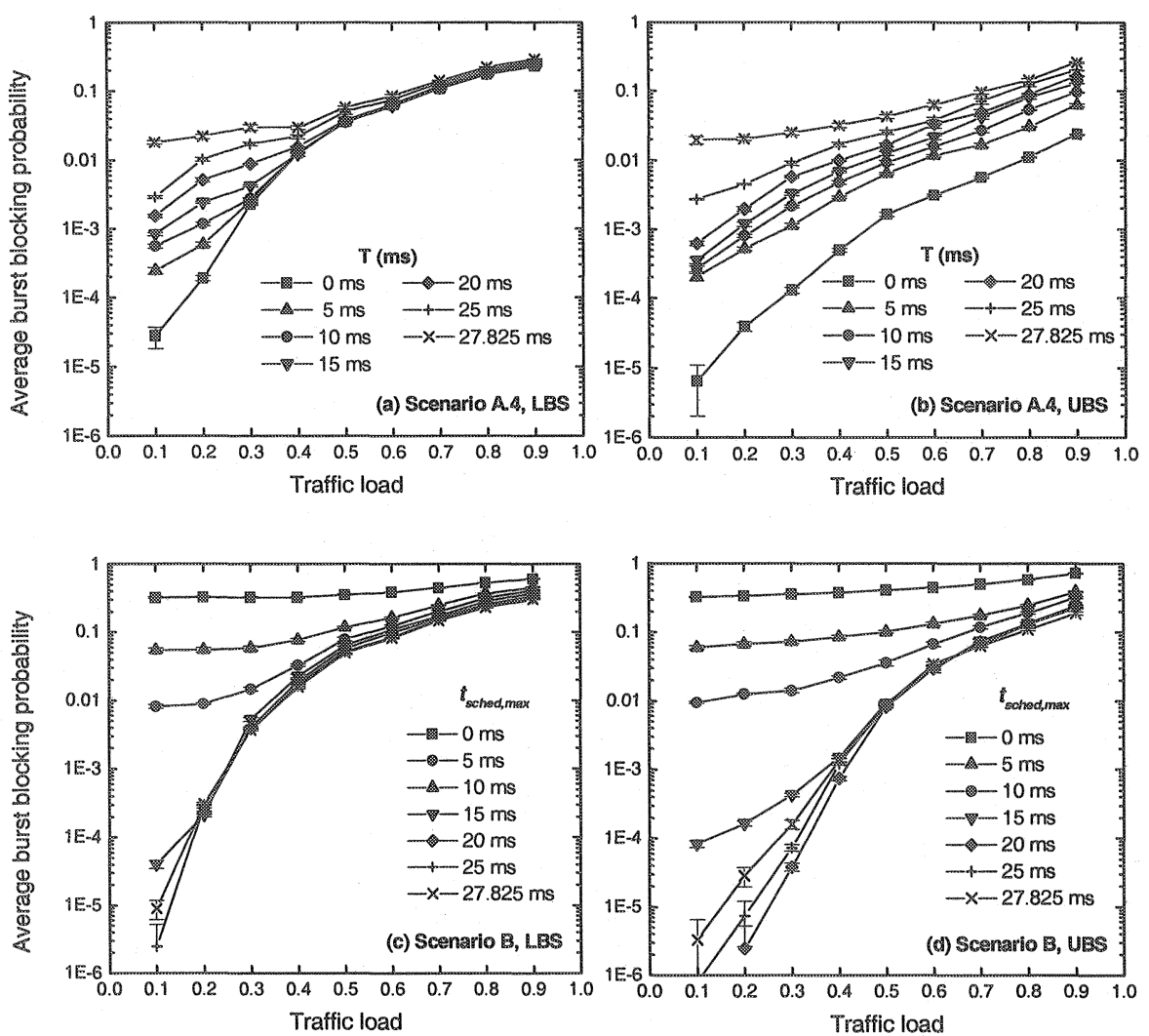

Figure 2. Average burst blocking probability for the NSFNet configured as a WR-OBS with centralised control and 12 wavelengths. A maximum end-to-end delay of $90 \mathrm{~ms}$ is guaranteed for all the packets. (a) Results for scenario A.4 and LBS, (b) results for scenario A.4 and UBS, (c) results for scenario $B$ and LBS, (d) results for scenario $B$ and UBS.

Note that the lowest blocking probability is not obtained with the highest possible $t_{\text {sched,max. }}$. This is because there is a trade-off between $T$ and $t_{\text {sched,max }}$. When $T$ is increased, lightpaths are more efficiently used (the overhead due to lightpath set-up is less significant as lightpaths are held longer, since the aggregation time, and hence the amount of data to transmit, increase), and this leads to a decrease on blocking probability. When $t_{\text {sched,max }}$ is increased the available time for reattempting a connection increases, and hence the blocking probability decreases. Since the aim is to provide an upper-bounded end-to-end delay, both parameters cannot be simultaneously increased. If $T$ is increased, then $t_{\text {sched,max }}$ must decrease, and vice versa. For that reason, there is an optimal combination of $T$ and $t_{\text {sched,max. }}$. 
Again, UBS obtains better results than LBS. When using this scenario and the UBS aggregation method, the network can carry a traffic load of up to 0.35 while satisfying the QoS requirements.

As shown in Figure 2.d, the optimal $t_{\text {sched,max }}$ for NSFNet is $20 \mathrm{~ms}$, so $T+$ $\Delta_{p h, s d \text { min }}=68.8 \mathrm{~ms}$. Therefore, timers in the network should be set to values between $T=7.825 \mathrm{~ms}$ and $T=67.225 \mathrm{~ms}$. (The former timer is associated to the buffer for transmission from CA2 to NY, and the latter is associated to transmission from PA to NJ, and from PA to NY.)

\subsubsection{Comparison of the scenarios}

Figure 3 shows the best value in terms of blocking probability for all the strategies proposed. The blocking probability increases with the traffic load, but strategies A.1 to A.3 have a local minimum around 0.7 and 0.8 , which can be due to unfairness issues. The lowest blocking probabilities are obtained for scenario B for low traffic loads, and for scenario A.4 for higher loads.

To study network fairness in each modelled scenario, we study the blocking probabilities associated to each $s-d$ pair. In this section we only show the results for strategies A.3, A.4 and B. We have assumed again a maximum end-to-end delay of $90 \mathrm{~ms}$ for all the packets, and the traffic load has been set in order to give a global blocking probability around $10^{-2}$. UBS has been used as aggregation method, adjusting timers to the optimum value according to Figures 2.b and 2.d $(T=0 \mathrm{~ms}$ for A.3 and A.4, and and $T+$ $\Delta_{p h, s d \text {,min }}=68.8 \mathrm{~ms}$ for scenario B). Figure 4 shows scatter diagrams of the average burst blocking probability for each $s-d$ pair versus the minimum location-dependent delay.

When applying scenario A.3, the blocking probability is heavily dependent on $\Delta_{p h, s d \text { min }}$. Scenarios A.4 and B have blocking probabilities which are approximately uncorrelated with $\Delta_{p h, s d, \text { min }}$. Better results on fairness are obtained with strategy $B$ as blocking probabilities are more concentrated around the average - solid lines in Figure 4- with this strategy (the coefficient of variation is lower). Anyway, the network is not fair yet as different pairs have different blocking probabilities. The proposed methods take into account distance information, but there are other topological factors with an impact on fairness and these facts have not been considered. For instance, some links are more congested than others (which in turn depends on traffic statistics), and not all nodes have the same physical degree (number of in-going and out-going links). Therefore, although strategy B is a good starting point, further research should be carried out on this topic. 


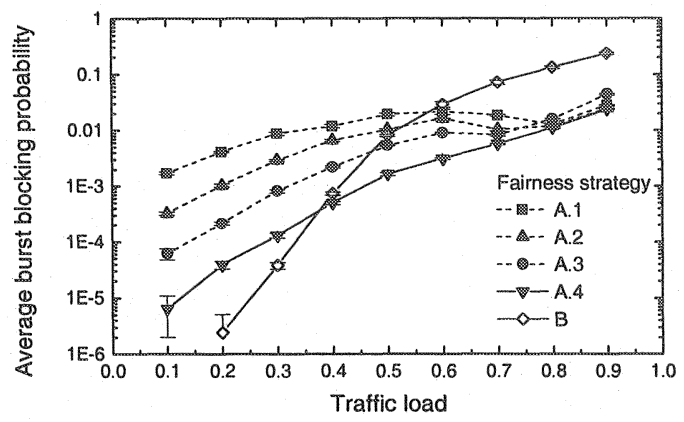

Figure 3. Average burst blocking probability for the NSFNet configured as a WR-OBS with centralised control and 12 wavelengths, when the UBS scheme is used. A maximum end-toend delay of $90 \mathrm{~ms}$ is guaranteed for all the packets. Different fairness strategies are used.
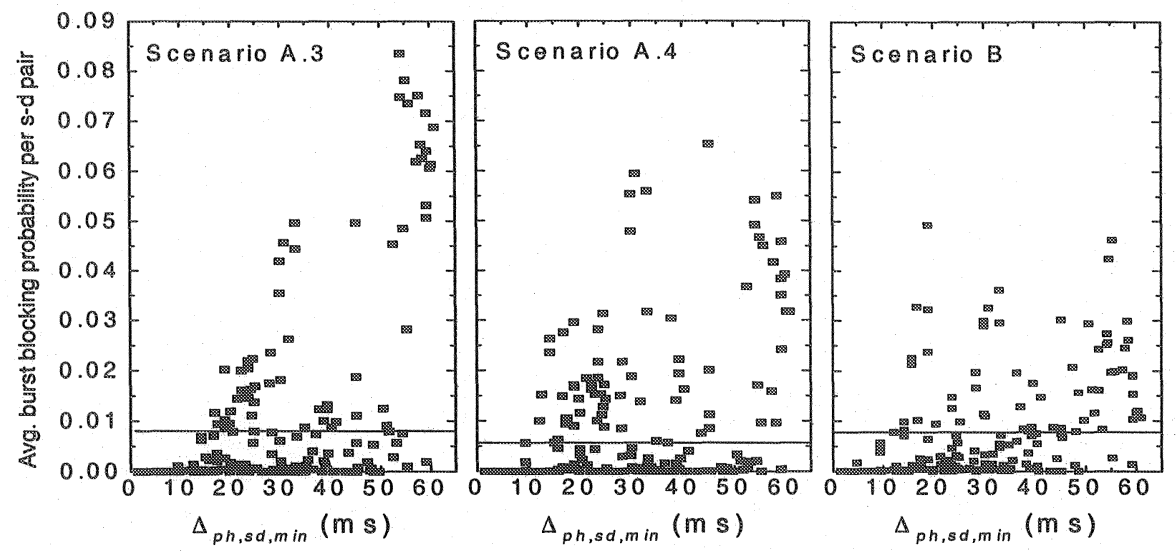

Figure 4. Scatter diagrams for the NSFNet configured as a WR-OBS with centralised control, 12 wavelengths, and UBS. The traffic load has been set to 0.6 for policy A.3, 0.7 for A.4, and 0.5 for $\mathrm{B}$. Solid lines show the global average burst blocking probability.

\subsection{Impact of the network diameter on the maximum supported traffic load}

To understand the impact of the network diameter on the maximum traffic load supported by a WR-OBS, the NSFNet topology, where the edges are scaled down by a factor of three, is analysed. Thus for the original and the scaled versions of the NSFNet the network diameter is $4455 \mathrm{~km}$ and $1485 \mathrm{~km}$, respectively. As UBS and scenario B have been shown to be most efficient in terms of blocking probability and fairness, these are used as a basis for the simulation in this section. As shown in Figure 5, a traffic load 
of up to nearly 0.7 can be carried by the network while satisfying the same QoS parameters than in previous sections.

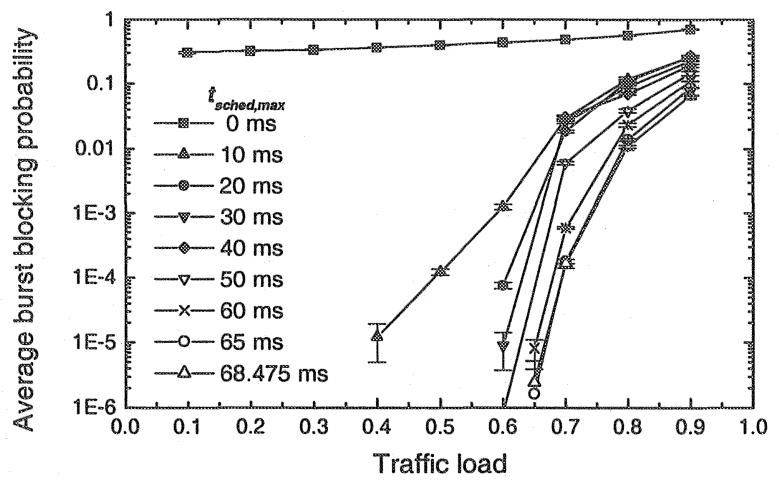

Figure 5. Average burst blocking probability for a scaled version of NSFNet (link distances divided by 3) configured as a WR-OBS with centralised control and 12 wavelengths, when the UBS scheme and policy B are used. $\Delta_{e e, \max } \leq 90 \mathrm{~ms}$ is guaranteed for all the packets.

Therefore, the scaled version can carry twice the traffic load that the original NSFNet could with the same guarantees. Hence, the lower the diameter of a network is, the greater the traffic range is in which the network has performance advantages when compared to a quasi-static WRON. There are two reasons for this behaviour. First of all, since $\Delta_{p h, s d, m i n}$ is lower in networks with smaller diameters, there is less overhead in lightpaths. As lightpaths are more efficiently used, a lower number of lightpaths will be simultaneously established, while carrying the same total traffic load, and so there will be more resources available [3]. Secondly, as the propagation delays are smaller but the maximum end-to-end delay does not vary, the maximum scheduling time can be higher, so there is more available time to reattempt a connection if a route and wavelength is not found the first time a request goes into the DRWA block.

\subsection{Impact of the number of wavelengths on the maximum supported traffic load}

Figure 6 shows the trade-off between the number of wavelengths available in a WR-OBS and the blocking probability (again, using UBS and scenario $B$ ), for the scaled version of NSFNet. The figure also illustrates one of the advantages of WR-OBS architectures: their potential for capacity upgrades. When the network is deployed, a few wavelengths may be sufficient for the routing of existing traffic. For instance, with 8 wavelengths 
a load of 0.35 can be transported in the example. However, if the traffic load increases, the system can be upgraded by adding wavelengths as required in order to accommodate the increase. For instance, a load of around 0.55 could be carried if 2 more wavelengths are added. Note that if NSFNet were configured as a static WRON, 13 wavelengths would be required in order to provide single-hop connectivity between all $s-d$ pairs, even if a low traffic load is to be transmitted. Hence, a WR-OBS could be attractive for network operators as the architecture can evolve with varying traffic demands.

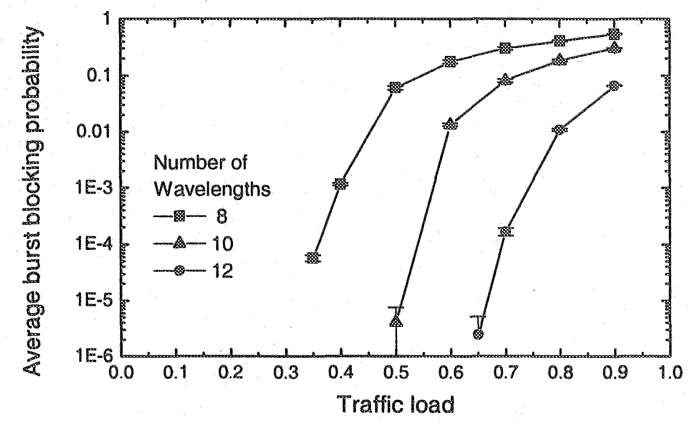

Figure 6. Average burst blocking probability for the scaled version of NFSNet (link distances divided by 3) configured as a WR-OBS with centralised control, using UBS, policy $B$, and for different numbers of wavelengths. $\Delta_{\mathrm{ee}, \max } \leq 90 \mathrm{~ms}$ is guaranteed for all the packets.

\section{SUMMARY}

Wavelength-routed optical burst-switched (WR-OBS) networks are a promising architecture to provide guaranteed latencies. In this paper, we have extended our previous work on scheduling policies in architectures with centralised control [6], to ensure bounded end-to-end delays in realistic networks, considering the asymmetry in terms of propagation delays between different $s-d$ pairs. In WR-OBS, as in any network with dynamic allocation of lightpaths, it is important not only to achieve low blocking probabilities, but also to provide a fair service independently of the location of the nodes. Hence, we have proposed a number of techniques to provide faimess so that all $s-d$ pairs have approximately the same (low) blocking probability due to exceeding the maximum allowable end-to-end delay. The best performing scenario has proved to be one in which timers controlling submission of requests to the control node are adjusted by taking into account both the distance to the control and to the destination node. It should be noted that this method did not consider other parameters with an impact 
on fairness (such as the physical degree of the nodes and link congestion, which in turn depends on traffic statistics), and requires further analysis.

Systematic comparison of the burst aggregation schemes and techniques for setting timers have been carried out. As a result of this, UBS has been validated as the optimal burst aggregation method in WR-OBS architectures, although it is less predictable on the wavelength-use time.

A realistic network, the NSFNet, has been analysed to judge whether it brings advantages when it is configured as a WR-OBS. Strict requirements on end-to-end delay (bounded by $90 \mathrm{~ms}$ ) and on blocking probability $\left(10^{-4}\right)$ were set. It was shown that under these conditions, a traffic load of 0.35 can be transmitted using 12 wavelengths. But if a lower number of wavelengths is used, say 10, the maximum traffic load that can be transmitted by the network decreases to only 0.01 . If the NSFNet is configured as a WRON, it can support higher traffic loads (up to 1) with only 13 wavelengths. Hence, a WR-OBS may not bring resource reduction in terms of wavelength requirements compared to a WRON. The reason for this has been related to the network diameter $(4455 \mathrm{~km})$, which introduces a high overhead in setting up of lightpaths. As the network diameter decreases, WR-OBS networks become more efficient when compared to WRONs. When a scaled version of the NSFNet topology was analysed (configured as a WR-OBS), it was shown that higher traffic loads could be transmitted than in the not-scaled one while using a lower number of wavelengths, together with a capacity to accommodate potential traffic growth. For instance, it allows a load of up to 0.35 with 8 wavelengths, and up to 0.7 with 12 wavelengths.

A key factor on the suitability of WR-OBS architectures is the traffic load that the network must support. According to [16], in current networks most links are utilised below $10 \%$ and the utilisation is expected to continue being low due to the improvements on link speed. Therefore, networks operate in the low traffic loads range, and it is in this regime where WR-OBS offers greatest operational benefits compared to WRONs. Moreover, a WR-OBS network can be easily upgraded by adding wavelengths as required in order to accommodate traffic increases.

Although this work has focused in centralised architectures, it is worth noting that distributed control in WR-OBS is also possible, and is preferred in helping maximise network resilience.

\section{ACKNOWLEDGMENTS}

The authors thank M. Düser and A. Zapata (UCL), Dr. D. Wischik (Univ. of Cambridge), F. González, J.C. Aguado and Prof. E.J. Abril (Univ. of Valladolid) for invaluable comments. Dr. C. Alberola (Univ. of Valladolid) 
allowed us to use some of his computers to launch simulations. The financial support from the Royal Society and the EU-IST WINMAN project is gratefully acknowledged. E. Kozlovski is a recipient of a UCL Graduate School Scholarship (1998-2001) and ORS (1999-2001) awards, as well as an award from the Ian Karten Charitable Trust (1998-9).

\section{REFERENCES}

[1] M. Düser, E. Kozlovski, R.I. Killey, P. Bayvel, "Design trade-offs in optical burst switched networks with dynamic wavelength allocation," Proc. ECOC 2000, pp. 23-24

[2] M. Düser, P. Bayvel, "Bandwidth utilisation and wavelength re-use in WDM Optical Burst-Switched Packet Networks," Proc. ONDM 2001, Vol. 1, 2001

[3] I. de Miguel, M. Düser, P. Bayvel, "Traffic load bounds for optical burst-switched networks with dynamic wavelength allocation," Proc. ONDM 2001, Vol. 1, 2001

[4] C. Qiao, M. Yoo, "Optical burst switching (OBS) - A new paradigm for an optical Internet," J. of High-Speed Networks, Vol. 8, 1999, pp. 69-84

[5] J.S. Tumer, "Terabit burst switching," J. of High-Speed Networks, Vol. 8, 1999, pp. 3-16

[6] E. Kozlovski, M. Düser, I. de Miguel, P. Bayvel, "Analysis of burst scheduling for dynamic wavelength assignment in optical burst-switched networks," Proc. IEEE LEOS 2001 Annual Meeting, paper TuD2

[7] 1. de Miguel, M. Düser, P. Bayvel, "The impact of dynamic wavelength assignment and burst aggregation in optical burst-switched networks," Proc. LCS 2001, pp. 167-170

[8] S. Baroni, P. Bayvel, "Wavelength requirements in arbitrarily connected wavelengthrouted optical networks," J. of Lightwave Technol., Vol. 15, Feb. 1997, pp. 242-251

[9] T.E. Stern, K. Bala, Multiwavelength optical networks: A layered approach, Prentice Hall PTR, 2000.

[10] Y. Xiong, M. Vandenhoute, H.D. Cankaya, "Control architecture in optical burstswitched WDM networks," IEEE J. on Selected Areas Commun., Vol. 18, Oct. 2000, pp. 1838-1851

[11] J. Liebeherr, D.E. Wrege, D. Ferrari, "Exact admission control for networks with a bounded delay service," IEEE/ACM Trans. on Networking, Vol. 4, Dec. 1996, pp. 885-901

[12] M. Baldi, Y. Ofek, "End-to-end delay analysis of videoconferencing over packetswitched networks," IEEE/ACM Trans. on Networking, Vol. 8, Aug. 2000, pp. 479-492

[13] A. Mokhtar, M. Azizoglu, "Adaptive wavelength routing in all-optical networks," IEEE/ACM Transactions on Networking, Vol. 6, Apr. 1998, pp. 197-206

[14] E. Hyytia, J. Virtamo, "Dynamic routing and wavelength assignment using first policy iteration," Proc. ISCC 2000, pp. 146-151

[15] S. Baroni, Routing and wavelength allocation in WDM optical networks, PhD Thesis, University College London, May 1998, pp. 118

[16] N. McKeown, "Do optics and routers belong together?," Keynote address, Opticomm 2001, Denver, Aug. 2001 (http://tiny-tera.stanford.edu/ nickm/talks/opticomm_2001.ppt) 